\title{
Perspectives of TFD on String Theory
}

\author{
M. C. B. Abdalla*, A. L. Gadelha and Daniel L. Nedel
}

Instituto de Física Teórica, Unesp, Pamplona 145, São Paulo, SP, 01405-900, Brazil

E-mail: mabdalla@ift.unesp.br, gadelha@ift.unesp.br,

danieleift.unesp.br

Considering the type IIB superstring in a pp wave background some recent ideas and perspectives of Thermo Field Dynamics on string theory are presented. The thermal Fock space is constructed attempting to consider a possible finite temperature version of the BMN correspondence in this framework. Also, the thermal vacuum is identified as a string boundary state realizing the thermal torus interpretation in the ambit of Thermo Field Dynamics. Such a interpretation consists of a generalization of some recent analysis for the closed bosonic string.

Fourth International Winter Conference on Mathematical Methods in Physics 09 - 13 August 2004

Centro Brasileiro de Pesquisas Fisicas (CBPF/MCT), Rio de Janeiro, Brazil

\footnotetext{
${ }^{*}$ Speaker.

${ }^{\dagger}$ M. C. B. A. was partially supported by the CNPq Grant 302019/2003-0, A. L. G. and D. L. N. are supported by a FAPESP post-doc fellowship.
} 
For the last 20 years, string theory has been considered the best candidate for a theory that quantizes gravity. The perturbative sector of the theory was explored in order to place the YangMills and gravitation theory at the same footing. The most impressive characteristic of string theory comes from the fact that the structure of the pertubative string theory is much more constrained than the one of field theory. This constrained structure gives the dimension of the space time, the gauge group and demands supersymmetry. Also, the formulation of the perturbative string at finite temperature is constrained. There is a temperature at above which the statistical partition function diverges (Hagedorn temperature) [1]. The very existence of the Hagedorn temperature shows that the fundamental degrees of freedom of the string theory could not be the ones of the perturbative string. In this direction, the perturbative string theory at finite temperature may provides important pieces of evidence of the true degrees of freedom of the theory at non-perturbative regime (higher temperatures). However, an understanding of the Hagedorn temperature, and the implications of this constrain in the space-time physics is still lacking. This lack of understanding motivates the study of new methods to introduce temperature in string theory. For example, it is known that the Hagedorn temperature comes from the exponential growth of states as function of energy. In this case the passage of the microcanonical ensemble to gran-canonical ensemble of statistical mechanics is absolutely non-trivial and it is hard to see where the results of one formalism is valid in the other. In this sense a more general formalism to deal with systems at finite temperature could be very useful. It is the case of the formalism that is going to be presented here, named Thermo Field Dynamics (TFD). The TFD was developed by Takahashi and Umezawa in order to handle finite temperature with a real time operator formalism [2]. The main idea is to interpret the statistical average of an operator $Q,\langle Q\rangle$ as the expectation value in a thermal vacuum

$$
\langle Q\rangle=\langle 0(\beta)|Q| 0(\beta)\rangle
$$

for $\beta=1 / T$ where $T$ is the temperature.

The thermal vacuum is constructed by means of a Bogoliubov transformation. Also, the creation and annihilation operators are transformed in order to construct a thermal Fock space. All the thermodynamics quantities can be defined as matrix elements of an operator in the thermal vacuum. The thermal effects are consequence of the condensed state structure of that thermal vacuum.

Concerning string theory, the idea of building a thermal Fock space can be very fruitful. For example, the "coherent state" description of $D_{p}$-branes in the perturbative limit of the theory, where they are viewed as a boundary state constructed in the Fock space of the closed string [3], made such kind of approach particularly tempting. In fact, in this context, the TFD has been used in order to study thermal properties of bosonic $D_{p}$-branes $[4,5,6,7,8,9]$. Another important example rests in the ADS/CFT correspondence. More specifically when one wants to study thermodynamics of type IIB superstring propagating in a pp wave background. In this case, the operatorial characteristic of TFD is suitable [10], since the so called Berenstein-Maldacena-Nastase (BMN) correspondence [11] can be explored at finite temperature directly in the Fock space.

In this work, some recent ideas and perspectives of the TFD approach on string theory are presented concentrating on the study of type IIB superstring in a pp-wave background at finite temperature. This system is worked on in order to present some perspective on the understanding of a thermal BMN correspondence and at the end an interpretation for the thermal vacuum is made in terms of a particular boundary state for the system under consideration. This interpretation allows 
one to understand the relationship between imaginary time [12] and TFD when both formalisms are used to study type IIB superstring at finite temperature. It consists of a generalization, to include fermionic degrees of freedom in a recent analysis for the closed bosonic string [13].

In the last years the BMN conjecture [11], was explored to give some insights into the strings thermodynamics in terms of Yang Mills thermodynamics $[14,15]$. In this conjecture the string propagates in a pp wave background. Such a background is obtained as the Penrose limit of $A D S_{5} \times$ $S^{5}$ [16], where the only surviving components of the Ramond-Ramond five form are: $F_{+1234}=$ $F_{+5678}=\mu$. On the gauge theory side the limit focuses on a set of operators which have R charges $J$ and the conformal dimension $\Delta$ satisfying $J \approx \sqrt{N}$ and $\Delta \approx J$, for fixed Yang-Mills coupling and $N$ going to infinity. This set up provides a complete dictionary between self-states of the string hamiltonian and Yang-Mills operators with $\Delta-J$ charge. For example, the vacuum in the string side has zero energy and it is related to an operator in the gauge side with zero value for $\Delta-J$

$$
\left|0, p^{+}\right\rangle \rightarrow O^{J}(0)|v a c\rangle, \quad O(x)=\frac{1}{\sqrt{J N^{J}}} \operatorname{Tr} Z^{J},
$$

where $|v a c\rangle$ is the Yang-Mills vacuum and $O^{J}$ is composed of two out of the six scalar fields of the $\mathcal{N}=4$ super Yang Mills multiplet: $Z=\frac{1}{2}\left(\phi^{5}+i \phi^{6}\right)$. The trace is taken over the $S U(N)$ index. Lately there have been some interesting works studying finite temperature effects of type IIB superstring in the pp waves background $[17,18,19]$. In a general way these works used the imaginary time formalism, to compute the superstring partition function and the free energy on a torus. However, using this formalism is difficult to take into account in the statistical average, only sectors that survive to the Penrose limit. As a consequence it is hard to see if the BMN correspondence works at finite temperature. On the order hand, with the TFD approach it is possible to construct a thermal Fock space for both sides of the conjecture and verify directly in this Fock space if the dictionary (2) works.

The solutions of the equations of motion with periodic boundary conditions for the type II superstring in pp wave background are [20]

$$
\begin{array}{r}
X^{I}=x_{0}^{I} \cos (m \tau)+\frac{\alpha^{\prime}}{m} p_{0}^{I} \sin (m \tau)+\sqrt{\frac{\alpha^{\prime}}{2}} \sum_{n>0} \frac{1}{\sqrt{\omega_{n}}}\left[\left(a_{n}^{I} e^{-i\left(\omega_{n} \tau-k_{n} \sigma\right)}+a_{n}^{\dagger I} e^{i\left(\omega_{n} \tau-k_{n} \sigma\right)}\right)\right. \\
\left.+\left(\bar{a}_{n}^{I} e^{-i\left(\omega_{n} \tau+k_{n} \sigma\right)}+\bar{a}_{n}^{\dagger I} e^{i\left(\omega_{n} \tau+k_{n} \sigma\right)}\right)\right],
\end{array}
$$

and

$$
\begin{array}{r}
S^{a}=\cos (m \tau) S_{0}^{a}+\sin (m \tau) \Pi_{a b} \bar{S}_{0}^{b}+\sum_{n>0} c_{n}\left[S_{n}^{a} e^{-i\left(\omega_{n} \tau-k_{n} \sigma\right)}+S_{n}^{\dagger a} e^{i\left(\omega_{n} \tau-k_{n} \sigma\right)}\right. \\
\left.+i \frac{\omega_{n}-k_{n}}{m} \Pi_{a b}\left(\bar{S}_{n}^{b} e^{-i\left(\omega_{n} \tau+k_{n} \sigma\right)}-\bar{S}_{n}^{\dagger b} e^{i\left(\omega_{n} \tau+k_{n} \sigma\right)}\right)\right], \\
\bar{S}^{a}=\cos (m \tau) \bar{S}_{0}^{a}-\sin (m \tau) \Pi_{a b} S_{0}^{b}+\sum_{n>0} c_{n}\left[\bar{S}_{n}^{a} e^{-i\left(\omega_{n} \tau+k_{n} \sigma\right)}+\bar{S}_{n}^{\dagger a} e^{i\left(\omega_{n} \tau+k_{n} \sigma\right)}\right. \\
\left.-i \frac{\omega_{n}-k_{n}}{m} \Pi_{a b}\left(S_{n}^{b} e^{-i\left(\omega_{n} \tau-k_{n} \sigma\right)}-S_{n}^{\dagger b} e^{i\left(\omega_{n} \tau-k_{n} \sigma\right)}\right)\right],
\end{array}
$$


where we set $m=\mu \alpha^{\prime} p^{+}$and

$$
\omega_{n}=\sqrt{m^{2}+k_{n}^{2}}, \quad c_{n}=\frac{1}{\sqrt{1+\left(\frac{\omega_{n}-k_{n}}{m}\right)^{2}}}, \quad k_{n}=2 \pi n .
$$

The canonical quantization gives the standard commutator and anti-commutator relations of harmonic oscillator and the zero mode part is written as follows

$$
\begin{aligned}
a_{0}^{I} & =\frac{1}{\sqrt{2 m}}\left(p_{0}^{I}-i m x_{0}^{I}\right), \quad a_{0}^{\dagger I}=\frac{1}{\sqrt{2 m}}\left(p_{0}^{I}+i m x_{0}^{I}\right), \\
S_{ \pm}^{a} & =\frac{1}{2}(1 \pm \Pi)_{a b} \frac{1}{\sqrt{2 m}}\left(S_{0}^{b} \pm i \bar{S}_{0}^{b}\right), \quad S_{ \pm}^{\dagger a}=\frac{1}{2}(1 \pm \Pi)_{a b} \frac{1}{\sqrt{2 m}}\left(S_{0}^{b} \mp i \bar{S}_{0}^{b}\right) .
\end{aligned}
$$

The vacuum $\left|0, p^{+}\right\rangle$is defined by

$$
\begin{aligned}
S_{n}\left|0, p^{+}\right\rangle=\bar{S}_{n}\left|0, p^{+}\right\rangle=0, & n>0, \\
a_{n}^{I}\left|0, p^{+}\right\rangle=\bar{a}_{n}^{I}\left|0, p^{+}\right\rangle=0, & n>0, \\
S_{ \pm}\left|0, p^{+}\right\rangle=a_{0}^{I}\left|0, p^{+}\right\rangle=0 . &
\end{aligned}
$$

Let us to introduce the temperature in this system using TFD. The TFD algorithm starts by duplicating the degrees of freedom. To this end a copy of the original Hilbert space is introduced and denoted by $\widetilde{H}$. The tilde Hilbert space is built with a set of oscillators: $\tilde{a}_{0}, \tilde{S}_{ \pm}, \tilde{a}_{n}, \tilde{\bar{a}}_{n}, \tilde{S}_{n}, \tilde{\bar{S}}_{n}$ that have the same (anti-) commutation properties as the original ones. The operators of the two systems (anti-) commute among themselves and the total Hilbert space is the tensor product of the two spaces. The map between the tilde and non-tilde operators is defined by the following tilde (or dual) conjugation rules $[2,21]$

$$
\begin{aligned}
\left(A_{i} A_{j}\right) & =\widetilde{A}_{i} \widetilde{A}_{j}, \quad\left(A_{i}^{\dagger}\right),=\sigma\left(\widetilde{A}_{i}\right)^{\dagger}, \quad\left(\widetilde{A}_{i}\right)=A_{i}, \\
\left(c A_{i}+A_{j}\right) & =c^{*} \sigma \widetilde{A}_{i}+\sigma \widetilde{A}_{j}, \quad\left[\widetilde{A}_{i}, A_{j}\right]=0,
\end{aligned}
$$

where $\sigma=1$ for bosons, $\sigma=-1$ for fermions and $c \in \mathbb{C}$. From these rules, the tilde system can be describe by a string that propagates backwards in imaginary time [13]. So, the TFD starts defining two independent free strings, defining two cylinders.

We can now construct the thermal vacuum. This is achieved by implementing a Bogoliubov transformation in the total Hilbert space. The transformation generator is given by

$$
\begin{gathered}
G=G^{B}+G^{F}, \\
G^{B}=G_{0}^{B}+\sum_{n=1}\left(G_{n}^{B}+\bar{G}_{n}^{B}\right), \\
G^{F}=G_{+}^{F}+G_{-}^{F}+\sum_{n=1}\left(G_{n}^{F}+\bar{G}_{n}^{F}\right),
\end{gathered}
$$

where

$$
G_{0}^{B}=-i \theta_{0}^{B}\left(a_{0} \cdot \tilde{a}_{0}-\tilde{a}_{0}^{\dagger} \cdot a_{0}^{\dagger}\right)
$$




$$
\begin{aligned}
G_{n}^{B} & =-i \theta_{n}^{B}\left(a_{n} \cdot \tilde{a}_{n}-\tilde{a}_{n}^{\dagger} \cdot a_{n}^{\dagger}\right), \\
\bar{G}_{n}^{B} & =-i \bar{\theta}_{n}^{B}\left(\bar{a}_{n} \cdot \tilde{\bar{a}}_{n}-\tilde{\bar{a}}_{n}^{\dagger} \cdot \bar{a}_{n}^{\dagger}\right), \\
G_{ \pm}^{F} & =-i \theta_{ \pm}^{F}\left(\widetilde{S}_{ \pm} \cdot S_{ \pm}-S_{ \pm}^{\dagger} \cdot \widetilde{S}_{ \pm}^{\dagger}\right), \\
G_{n}^{F} & =-i \theta_{n}^{F}\left(\widetilde{S}_{n} \cdot S_{n}-S_{n}^{\dagger} \cdot \widetilde{S}_{n}^{\dagger}\right), \\
G_{n}^{F} & =-i \bar{\theta}_{n}^{F}\left(\widetilde{\bar{S}}_{n} \cdot \bar{S}_{n}-\bar{S}_{n}^{\dagger} \cdot \widetilde{\bar{S}}_{n}^{\dagger}\right) .
\end{aligned}
$$

Here, the $B$ and $F$ labels specify fermions and bosons, the dots represent the inner products and $\theta, \bar{\theta}$ are parameters that depend on temperature. The thermal vacuum is defined by the following relation

$$
\left.|0(\theta)\rangle=e^{-i G}|0\rangle\right\rangle
$$

The effect of the Bogoliubov transformation is to entangle the elements of the two Hilbert spaces. After the transformation, the image of the two independent strings is lost. The creation and annihilation operators at $T \neq 0$ are given by the Bogoliubov transformation as follows

$$
\begin{aligned}
& a_{n}^{I}\left(\theta_{n}\right)=e^{-i G} a_{n}^{I} e^{i G}=\cosh \left(\theta_{n}\right) a_{n}^{I}-\sinh (\theta) \widetilde{a}_{n}^{\dagger I}, \\
& S_{n}^{a}\left(\theta_{n}\right)=e^{-i G} S_{n}^{a} e^{i G}=\cos \left(\theta_{n}\right) S^{a}{ }_{n}-\sin \left(\theta_{n}\right) \widetilde{S}_{n}^{\dagger a},
\end{aligned}
$$

and the same for the other operators. These operators annihilate the state written in (19) defining it as the vacuum. The thermal Fock space is constructed by applying the thermal creation operators to the vacuum (19). As the Bogoliubov transformation is canonical, the thermal operators obey the same commutation relations as the operators at $T=0$.

Next, we show how the thermodynamical quantities can be derived introducing first a free energy like potential defined by

$$
\mathcal{F}=\mathcal{E}-\frac{1}{\beta} \mathcal{S}
$$

where $\mathcal{E}$ is related with the thermal energy and $\mathcal{S}$ with the entropy of the string. In TFD the thermal energy is given by computing the matrix elements of the $T=0$ hamiltonian in the thermal vacuum. In order to take into account the level matching condition, the shifted hamiltonian is used

$$
\mathcal{E}=\left\langle 0(\theta)\left|H_{S}\right| 0(\theta)\right\rangle=\left\langle 0(\theta)\left|H+\frac{i \lambda}{\beta} P\right| 0(\theta)\right\rangle .
$$

Here $\lambda$ is a lagrange multiplier that fixes the $S^{1}$ isometry of the closed string and $P$ is the worldsheet momentum. The entropy of the superstring is calculated by evaluating the expected value of the entropy operator defined in [10] in the thermal vacuum. By minimizing the potential $\mathcal{F}$ with respect to $\theta$ we find the explicit dependence of these parameters in relation to $\omega_{n}, \beta$ and $\lambda$. In this way we have

$$
\sinh ^{2}\left(\theta_{0}^{B}\right)=\frac{1}{e^{\frac{\beta m}{p+}}-1}, \quad \sin ^{2}\left(\theta_{ \pm}^{F}\right)=\frac{1}{e^{\frac{\beta m}{p+}}-1},
$$

for the zero modes, and

$$
\begin{aligned}
\sinh ^{2}\left(\theta_{n}^{B}\right)=\frac{1}{e^{\frac{\beta \omega_{n}}{p+}+i \lambda k_{n}}-1}, & \sinh ^{2}\left(\bar{\theta}_{n}^{B}\right)=\frac{1}{e^{\frac{\beta \omega_{n}}{p+}-i \lambda k_{n}}-1} \\
\sin ^{2}\left(\theta_{n}^{F}\right)=\frac{1}{e^{\frac{\beta \omega_{n}}{p+}+i \lambda k_{n}}+1}, & \sin ^{2}\left(\bar{\theta}_{n}^{F}\right)=\frac{1}{e^{\frac{\beta \omega_{n}}{p+}-i \lambda k_{n}}+1},
\end{aligned}
$$


for the other modes. With these results, one can write the free energy like potential as [10]

$$
\mathcal{F}\left(\lambda, \beta / p^{+}\right)=-\frac{1}{\beta} \ln \prod_{n=\mathbb{Z}}\left[\frac{1+e^{-\frac{\beta \omega_{n}}{p+}+i \lambda k_{n}}}{1-e^{-\frac{\beta \omega_{n}}{p+}+i \lambda k_{n}}}\right]^{8}=-\frac{1}{\beta} \ln \left(z_{l c}\left(\beta / p^{+}, \lambda\right)\right),
$$

where $z_{l c}\left(\beta / p^{+}, \lambda\right)$ is the torus transverse partition function calculated in [17].

So, the thermal vacuum reproduces the same results of the imaginary time formalism, when the worldsheet is defined on a torus with moduli space parameters $\tau=\lambda+i \tau \frac{\beta}{2 \pi}$. The next step would be to construct a thermal vacuum for the Yang Mills side and verify the BNM dictionary directly in the thermal Fock space. However, before going on with this program a question needs to be answered; How did the torus defined by the moduli space parameter $\tau=\lambda+i \tau \frac{\beta}{2 \pi}$ appears in the TFD approach?

The TFD approach starts with a tree level string and an auxiliary string (tilde system). Suppose we want to construct a torus with the two cylinders defined by the strings. As the partition function for the torus is defined after Wick rotation, we need to go to Euclidean time $(\tau=-i t)$. In addiction, the tilde string is a mirror of the string and propagates backwards in imaginary time. So, when the string propagates from zero to a euclidian time $\beta / 2$ for example, the tilde string propagates from zero to $-\beta / 2$ in euclidian time. Then, a torus can be constructed by gluing together the end of the original cylinder with the origin of the tilde one, and vice-versa [13]. Also, before gluing, the identification $\tilde{\sigma}=\sigma-\pi \lambda$, must be done in order to take into account the Dehn twist in one cycle. The two parameters of the resulting torus moduli space will be related to $\beta$ and $\lambda$. The above considerations can be written as follows

$$
\begin{aligned}
X(t, \sigma)-\tilde{X}\left(-t-\frac{\beta}{2}, \sigma-\lambda \pi\right) & =0, \quad X^{I}\left(-\tilde{t}-\frac{\beta}{2}, \tilde{\sigma}+\lambda \pi\right)-\tilde{X}^{I}(\tilde{t}, \tilde{\sigma})=0, \\
S^{a}(t, \sigma)-\widetilde{S}^{a}\left(-t-\frac{\beta}{2}, \sigma-\lambda \pi\right) & =0, \quad S^{a}\left(-\tilde{t}-\frac{\beta}{2}, \tilde{\sigma}+\lambda \pi\right)-\widetilde{S}^{a}(\tilde{t}, \tilde{\sigma})=0 .
\end{aligned}
$$

Expanding $X(t, \sigma), S(t, \sigma)$ and $\widetilde{X}(\widetilde{t}, \widetilde{\sigma}), \widetilde{S}(t, \sigma)$ in modes, the above identification turns out to be a set of operatorial equations for a boundary state $|\Phi\rangle=|\phi, \widetilde{\phi}\rangle$. Resolving the boundary state equations we get the following normalized solution

$$
\begin{aligned}
|\Phi\rangle & =\left(\frac{1}{\cosh \left(\theta_{0}^{B}\right)}\right)^{8}\left(\cos \left(\theta_{+}^{F}\right)\right)^{4}\left(\cos \left(\theta_{-}^{F}\right)\right)^{4} e^{\tanh \left(\theta_{0}^{B}\right)\left(a_{0}^{\dagger} \cdot \tilde{a}_{0}^{\dagger}\right)} e^{\tan \left(\theta_{+}^{F}\right)\left(S_{+}^{\dagger} \cdot \tilde{S}_{+}^{\dagger}\right)+\tan \left(\theta_{+}^{F}\right)\left(S_{-}^{\dagger} \cdot \tilde{S}_{-}^{\dagger}\right)} \\
& \times \prod_{n=1}\left[\left(\frac{1}{\cosh \left(\theta_{n}^{B}\right)}\right)^{8}\left(\frac{1}{\cosh \left(\bar{\theta}_{n}^{B}\right)}\right)^{8} e^{\tanh \left(\theta_{n}^{B}\right)\left(a_{n}^{\dagger} \cdot \tilde{a}_{n}^{\dagger}\right)+\tanh \left(\bar{\theta}_{n}^{B}\right)\left(\bar{a}_{n}^{\dagger} \cdot \tilde{a}_{n}^{\dagger}\right)}\right. \\
& \times\left(\cos \left(\theta_{n}^{F}\right)\right)^{8}\left(\cos \left(\bar{\theta}_{n}^{F}\right)\right)^{8} e^{\left.\left.\tan \left(\theta_{n}^{F}\right)\left(S_{n}^{\dagger} \cdot \tilde{S}_{n}^{\dagger}\right)+\tan \left(\bar{\theta}_{n}^{F}\right)\left(\bar{S}_{n}^{\dagger} \cdot \tilde{\bar{S}}_{n}^{\dagger}\right)\right]|0\rangle\right\rangle .}
\end{aligned}
$$

This is precisely the thermal state (19) when the relations (25) are used and the Bogoliubov generators are expanded.

Finally, we can conclude that the effect of the Bogoliubov transformation is to entangle the two strings in order to make a torus in such away that the worldsheet fields are confined in a restricted region $\beta$ of the time axis. Such a confinement was pointed out in the scope of field theory and 
Casimir effect [22]. The thermal state is a boundary state responsible for this confinement. This is a very suggestive result, since it opens the possibility of other kinds of boundary states (D-branes for example) to appear at some higher temperature. It will be interesting to relate this topological interpretation for the thermal vacuum constructed for this type II superstring, to a thermal boundary state in the Yang Mills side of the BMN conjecture. This work is in progress. In other direction, we can use the operatorial method developed in [23] to study string at finite temperature on higher genus surfaces.

\section{References}

[1] J. J. Atick and E. Witten, The Hagedorn Transition And The Number Of Degrees Of Freedom Of String Theory, Nucl. Phys. B310 (1988) 291.

[2] Y. Takahasi and H. Umezawa, Thermo Field Dynamics, Collect. Phenom. 2 (1975) 55.

[3] P. Di Vecchia and A. Liccardo, D branes in string theory. I. NATO Adv. Study Inst. Ser. C. Math. Phys. Sci. 556 (2000) 1 [hep-th/9912161].

[4] I. V. Vancea, Bosonic D-branes at finite temperature, Phys. Lett. B487 (2000) 175 [hep-th/0006228].

[5] M. C. B. Abdalla, A. L. Gadelha and I. V. Vancea, Bosonic D-branes at finite temperature with an external field, Phys. Rev. D64 (2001) 086005 [hep-th/0104068].

[6] M. C. B. Abdalla, E. L. Graca and I. V. Vancea, Entropy of bosonic open string states in TFD approach, Phys. Lett. B536 (2002) 144 [hep-th/0201243].

[7] M. C. B. Abdalla, A. L. Gadelha and I. V. Vancea, On the SU(1,1) thermal group of bosonic strings and D-branes, Phys. Rev. D66 (2002) 065005 [hep-th/0203222].

[8] M. C. B. Abdalla, A. L. Gadelha and I. V. Vancea, TFD approach for bosonic strings and D(p)-branes, Int. J. Mod. Phys. A18 (2003) 2109 [hep-th/0301249].

[9] M. C. B. Abdalla, A. L. Gadelha and I. V. Vancea, D-branes at finite temperature in TFD, hep-th/0308114.

[10] D. L. Nedel, M. C. B. Abdalla and A. L. Gadelha, Superstring in a pp-wave background at finite temperature: TFD approach, Phys. Lett. B598 (2004) 121 [hep-th/0405258].

[11] D. Berenstein, J. M. Maldacena and H. Nastase, Strings in flat space and pp waves from $N=4$ super Yang Mills, JHEP 0204 (2002) 013 [hep-th/0202021].

[12] A. Das, Finite Temperature Field Theory, World Scientific, Singapore 1977.

[13] M. C. B. Abdalla, A. L. Gadelha and D. L. Nedel, Closed string thermal torus from thermofield dynamics, hep-th/0410068.

[14] B. R. Greene, K. Schalm and G. Shiu, On the Hagedorn behaviour of pp-wave strings and N $=4$ SYM theory at finite R-charge density, Nucl. Phys. B652 (2003) 105 [hep-th/0208163].

[15] R. C. Brower, D. A. Lowe and C. I. Tan, Hagedorn transition for strings on pp-waves and tori with chemical potentials, Nucl. Phys. B652 (2003) 127 [hep-th/0211201].

[16] M. Blau, J. Figueroa-O'Farrill, C. Hull and G. Papadopoulos, Penrose limits and maximal supersymmetry, Class. Quant. Grav. 19 (2002) L87 [hep-th/0201081].

[17] L. A. Pando Zayas and D. Vaman, Strings in RR plane wave background at finite temperature, Phys. Rev. D67 (2003) 106006 [hep-th/0208066]. 
[18] Y. Sugawara, Thermal amplitudes in DLCQ superstrings on pp-waves, Nucl. Phys. B650 (2003) 75 [hep-th/0209145].

[19] G. Grignani, M. Orselli, G. W. Semenoff and D. Trancanelli, The superstring Hagedorn temperature in a pp-wave background, JHEP 0306 (2003) 006 [hep-th/0301186].

[20] R. R. Metsaev and A. A. Tseytlin, Exactly solvable model of superstring in plane wave Ramond-Ramond background, Phys. Rev. D65 (2002) 126004 [hep-th/0202109].

[21] A. E. Santana, A. Matos Neto, J. D. M. Vianna, F. C. Khanna, Symmetry groups, density-matrix equations and covariant Wigner function, Physica A280 (2000) 405.

[22] J. C. da Silva, F. C. Khanna, A. Matos Neto and A. E. Santana, Generalized Bogoliubov transformation for confined fields: Applications in Casimir effect, Phys. Rev. A66 (2002) 052101 [hep-th/0208183].

[23] L. Alvarez-Gaume, C. Gomez, G. W. Moore and C. Vafa, Strings In The Operator Formalism, Nucl. Phys. B303 (1988) 455. 